\title{
DISTRIBUTED OBJECTS FOR PARALLEL NUMERICAL APPLICATIONS
}

\author{
Francoise Baude $^{1}$, Denis Caromel ${ }^{1}$ and David Sagnol ${ }^{1}$
}

\begin{abstract}
The $\mathrm{C}++/ /$ language (pronounced $\mathrm{C}++$ parallel) was designed and implemented with the aim of importing reusability into parallel and concurrent programming, in the framework of a MIMD model. From a reduced set of rather simple primitives, comprehensive and versatile libraries are defined. In the absence of any syntactical extension, the $\mathrm{C}++/ /$ user writes standard $\mathrm{C}++$ code. The libraries are themselves extensible by the final users, making $\mathrm{C}++/ /$ an open system. Two specific techniques to improve performances of a distributed object language such as $\mathrm{C}++/ /$ are then presented: Sharedon-Read and Overlapping of Communication and Computation. The appliance of those techniques is guided by the programmer at a very high-level of abstraction, so the additional work to yield those good performance improvements is kept to the minimum.
\end{abstract}

Mathematics Subject Classification. 68N15, 68N19.

Received: 11 December, 2001. Revised: 23 May, 2002.

\section{INTRODUCTION}

Reusability has been one of the major contributions of object-oriented programming; bringing it to parallel programming is one of our main goals, and a major step forward for software engineering of parallel systems. Part of the challenge is to combine the potential for extensive reuse with the high performance which is usually required of parallel and real-time systems.

Working mainly within the framework of physically distributed architectures, we are concerned with both explicit and implicit parallelism in both the problem and solution domains. Our applications include parallel data structures, computer-supported cooperative work (CSCW), and fault-tolerance and reliability in safetycritical and real-time systems.

To achieve this end, we began design and implementation of $\mathrm{C}++/ /$ early in 1994, and we are pursuing now this research in the context of the Java language, with the definition of a library called ProActive PDC [18]. The $\mathrm{C}++/ /$ language benefited from previous research done on the Eiffel// language [12,15]. Important ideas and techniques from that work have reappeared in the definition of a reduced set of simple primitives that are then composed to create comprehensive and versatile libraries, which - most importantly - can then be extended by end users.

Another important characteristic of our system is the complete absence of any syntactical extension to $\mathrm{C}++$. $\mathrm{C}++/ /$ users write standard $\mathrm{C}++$ code, relying on specific classes to give programs a parallel semantics. These

Keywords and phrases. Concurrency, data-driven synchronization, dynamic binding, inheritance, object-oriented concurrent programming, polymorphism, reusability, software development method, wait-by-necessity, overlap, object sharing.

1 OASIS, Joint Project CNRS, INRIA, University of Nice Sophia Antipolis, 2004 route des Lucioles,

BP 93, 06902 Valbonne Cedex, France. e-mail: Denis.Caromel@sophia.inria.fr

(C) EDP Sciences, SMAI 2002 
programs are then passed through a pre-processor, which generates new files. The original and new code is then compiled and linked with a standard $\mathrm{C}++$ compiler. When appropriate, all names related to the $\mathrm{C}++/ /$ system include the 11 root in their name (for "parallel"). During the presentation of our system, we will conform to the following symbols when introducing:

a model principle or rule: $\diamond$

a file used in our system:

a new syntax, class, or member: (S)

examples: $\nabla$

We hope these conventions ease reading and quick referencing through the paper.

This article begins by describing the basic features of our programming model, which is an MIMD model without shared memory. Section 3 deals with the control programming of processes, i.e. the definition of concurrent process activity. A recommended method for parallel programming in $\mathrm{C}++/ /$ is outlined in Section 4.1. Those parts of the programming environment which handle compilation and mapping are described in Section 4.2 , and an overview of the implementation techniques which make the system open and user-extensible is given in Section 4.3. Finally, we present two performance optimizations that to be applied demand some implication from the user, but at a high-level of abstraction: on one side, sharing objects among processes in case they are in the same address space, on the other side, overlapping communication with computation in the framework of remote method calls. This paper ends up with conluding remarks in Section 7.

\section{BASIC MODEL OF CONCURRENCY}

This section describes four important characteristics of our parallel programming model: parallel processes, communication between them, synchronization, and data sharing. As described below, we adopt a MIMD model without shared memory, which means that there are no directly-shared objects in our system.

Along with simplicity and expressiveness, reusability is one of our major concerns. More specifically, we want to allow users to take an existing $\mathrm{C}++$ system and transform it into a distributed one, so that they may derive parallel systems from sequential ones [14].

\subsection{Processes}

One of the key features of the object-oriented paradigm is the unification of the notions of module and type to create the notion of class. When adding parallelism, another unification is to bring together the concepts of class and process, so that every process is an instance of a class, and the process's possible behavior is completely described by its class.

$\diamond$ Model: the process structure is a class; a process is an object executing a prescribed behavior.

However, not all objects are processes. At run-time, we distinguish two kinds of objects: process objects (or active objects), which are active by themselves, with their own thread of control, and passive objects, which are normal objects. This second category includes all non-active objects. An example of the arrangement of processes and objects at run-time is given in Figure 1.

At the language level, there are two ways to generate active objects. In the first, an active object is obtained by instantiating a standard sequential $\mathrm{C}++$ class using Process_alloc:

(S) Syntax:

A* p; $\quad / / A$ is a normal sequential class

$\mathrm{p}=(\mathrm{A} *)$ new Process_alloc ( typeid $(\mathrm{A}), \ldots)$;

In this case, a standard sequential class A is instantiated to create an active object, which then has a FIFO synchronization: method invocations are serviced in the order in which they are made. The Process_alloc class is part of the $\mathrm{C}++/ /$ library, while typeid is the standard $\mathrm{C}++$ run-time type identification (RTTI) operator. We will refer to this technique as the allocation style of process creation, and say that it produces an allocation process, or allocation active object. The allocation style is convenient, but limited because it only allows us to create processes with a FIFO behavior. 


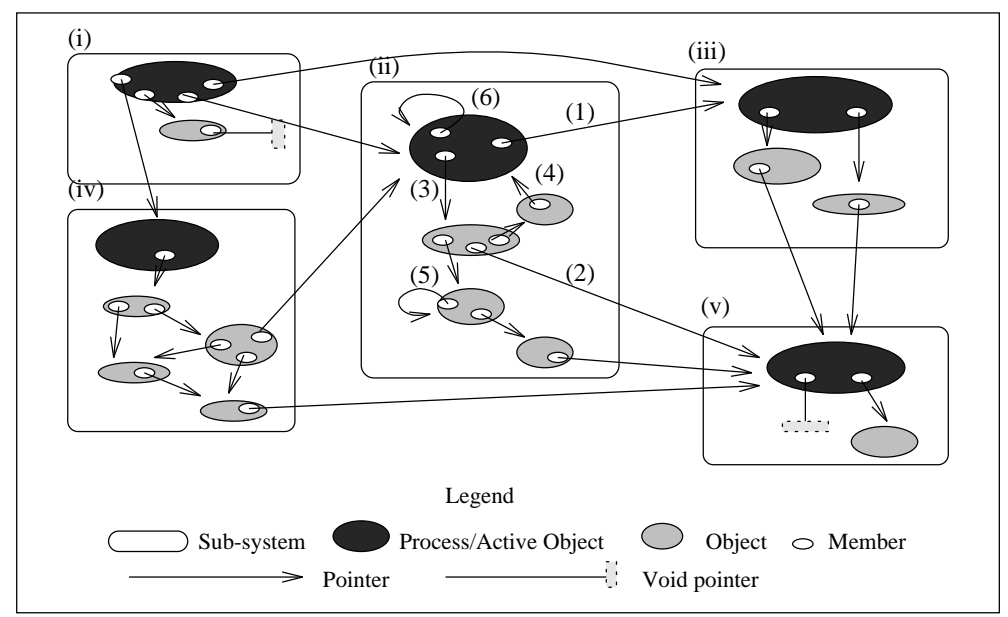

Figure 1. Processes and objects at run-time.

The second technique, which we call class-based, is more general:

$\diamond$ Model: all objects which are an instance of a class which publicly inherits from the Process class are processes.

This Process class is part of the $\mathrm{C}++/ /$ library. To use class-based process creation, the programmer must therefore derive a specific class, called a process class, from Process, as in:

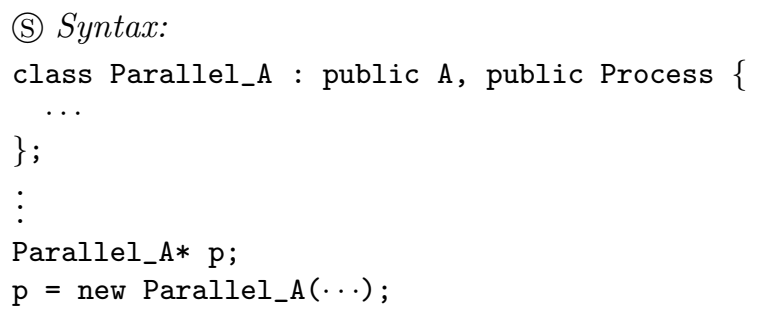

As with the allocation-based technique, instances of sub-classes of Process have a default FIFO behavior. However, as we will see in the following sections, it is possible to change this to create other behaviors. We say that the class-based technique generates class-based processes, or class-based active objects.

As shown in Figure 1, passive objects (i.e., objects which are not active) belong at run-time to a single process object. This organizes a parallel program into disjoint sub-systems, each of which consists of one active object encapsulating zero or more passive objects. Figure 2 presents the two styles of active object definition.

\subsection{Sequential or parallel processes}

A major design decision for any concurrent programming system is whether processes are sequential (i.e., single-threaded) or able to support internal concurrency (i.e., multi-threaded). Because our system is oriented towards reuse and software engineering of parallel systems, rather than operating systems programming, we made the following choice:

$\diamond$ Model: a process is sequential, it is single-threaded.

We believe that single-threaded processes are easier to reuse, and easier to write correctly.

The model does not allow the user to program multi-threaded processes, but this does not prevent multithreading at the operating system level. As we will see in Section 4.2, several sequential processes can be implemented with one multi-threaded operating system process for the sake of light-weightness. 


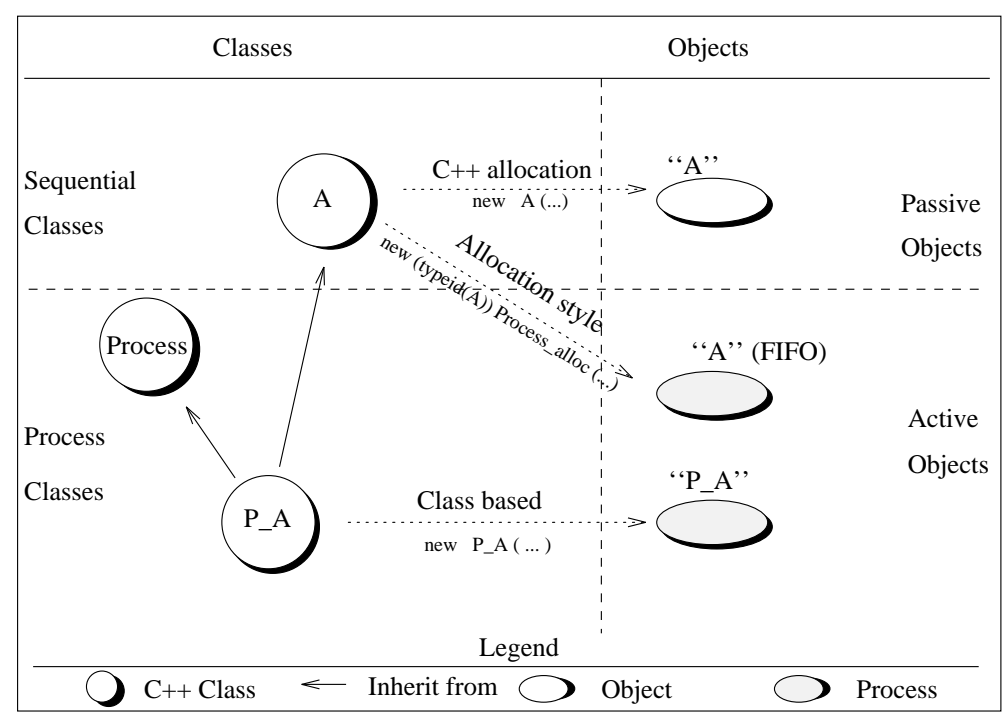

Figure 2. Allocation and class based active objects.

\subsection{Communication}

Since a process is an object, it has member functions. When an object owns a reference to a process, it is able to communicate with it by calling one of its public members. This is C++//'s inter-process communication (IPC) mechanism:

$\diamond$ Model: communications towards active objects appear syntactically programmed as member function calls.

The syntax of an IPC is unified with a standard call:

\section{(S) Syntax:}

$p \rightarrow f$ ( parameters );

This idea, introduced by the Actors model [3,30], means that what is sometimes called a process entry point is identical to a normal routine or member function.

While this idea is widely used in parallel object-oriented systems, there are many differences in the definition of the semantics of method-based IPC. In $\mathrm{C}++/ /$ :

$\diamond$ Model: communications are asynchronous between processes.

Function calls towards passive objects retain the synchronous semantics of standard $\mathrm{C}++$. This choice encourages parallel execution of objects, and makes each process code more independent and self-contained. As we will see further, it is also very important for supporting reusability. Synchronous function call is also possible in $\mathrm{C}++/ /$, but must be specified explicitly in either the function call or the process definition.

Systematic asynchronous IPC structures a C++// system into independent asynchronous sub-systems: all the communications between sub-systems are asynchronous. Figure 1 demonstrates five sub-systems.

\subsection{Synchronization}

Asynchronous communication can be difficult for programmers to manage. For instance, since even function calls to processes are asynchronous, before using result values one usually needs to explicitly add synchronizations in order to make sure they have been returned by the processes. Commonly, such models lack synchronization. We use a simple rule to address this drawback: wait-by-necessity. 
$\diamond$ Model: a process is automatically blocked when it attempts to use the result of a parallel member function call that has not yet been returned.

Thus, a caller does not wait for the result of an asynchronous function call until that value is explicitly used in some computation. Should a value not have been returned at that point, the caller is automatically blocked until the value becomes available. This mechanism implicitly synchronizes processes; the two primitives Wait and Awaited are provided for explicit synchronization.

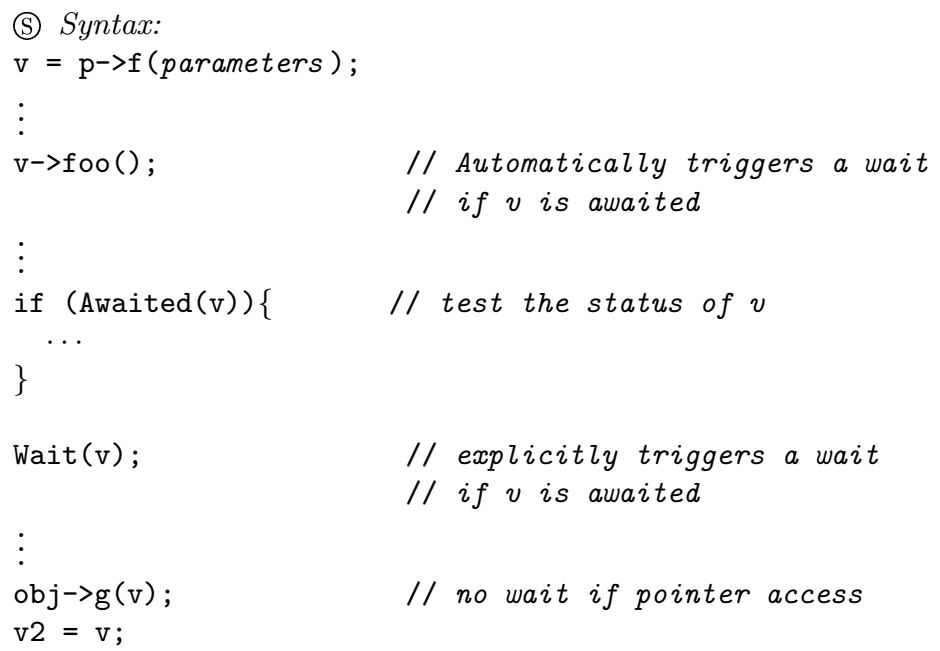

Program 1.1. Wait-by-necessity.

Program 1.1 summarizes the semantics of wait-by-necessity. The result of a function call not yet returned is called an awaited object. Our semantics define that no wait is triggered by assigning a pointer to such an object to another variable, or by passing such a pointer as a parameter. A wait occurs only when the program accesses the awaited object itself (which is syntactically a pointer access to the object) or transmits (copies) the object to another process.

Wait-by-necessity is a form of future [28], and is related to concepts found in several other languages: the Hurry primitive of Act1 [33], the CBox objects of ConcurrentSmalltalk [42], and the future type message passing of ABCL/1 [43]. However, an important difference is that the mechanism presented here is systematic and automatic, which is reflected in the absence of any special syntactic construction. This has a strong impact on reusability.

In order to avoid the run-time overhead involved in the implementation of wait-by-necessity, it is possible to avoid implicit synchronization and use the explicit synchronization primitives instead. This is a tradeoff between programming ease and reusability on one hand, and efficiency and speedup on the other.

\subsection{Sharing}

If two processes refer to the same object, method calls to that object may overlap, which raises all of the problems usually associated with shared data. To address this issue, each non-process object in $\mathrm{C}++/ /$ is a private object, and is accessible to only one process:

$\diamond$ Model: there are no shared passive objects.

We say that a private object belongs to its process's sub-system. The programming model ensures the absence of sharing:

$\diamond$ Model: the semantics of communication between processes is a copy semantics for passive objects. 
- A process is an active object, sequential and single-threaded.

- Communications between active objects are syntactically programmed as member function calls, and are asynchronous.

- Wait-by-necessity: a process is automatically synchronized, i.e. it waits, when it attempts to use the result of a member function call that has not been returned yet.

- There are no shared passive objects.

- The semantics of communication between processes is a copy semantics for passive objects.

FiguRE 3. Basic features of the $\mathrm{C}++/ /$ model.

All parameters are automatically transmitted by copy from one process to another. A deep copy of the object is achieved: when an object $\mathrm{o}$ is copied, all the objects referred to by pointers in o are deep copied as well. The implementation automatically and transparently handles the marshalling of data and pointers implied by this, as well as circular object structures:

(S) Syntax:

$\begin{aligned} \mathrm{p} \rightarrow \mathrm{f} \text { ( parameters ) ; } & / / \text { passive objects are automatically } \\ & \text { // passed by copy between processes. }\end{aligned}$

Processes, of course, are always transmitted by reference.

Figure 1 shows how shared objects do not appear in $\mathrm{C}++/ /$ programs. Each passive object is accessible to exactly one active object; each of the five sub-systems in this program consists of one active object and all the passive objects it can reach. The arcs labelled (1) and (2) are always activated as asynchronous communication (IPC), while the arcs labelled (3) to (6) are activated as normal function calls. As a consequence of the absence of shared objects, synchronization between sub-systems only occurs when one sub-system waits for a result value from another process.

Prohibiting shared data has also important methodological consequences. The absence of shared objects allows either an immediate reuse (through the automatic copy), or the identification of new processes to program in order to implement the shared objects. Finally, due to the absence of interleaving, it helps ensuring correctness of such parallel applications derived from sequential ones.

As we finished the basic characteristics of the programming model, Figure 3 summarizes them.

The model has some limitations: in order to be able to use polymorphism between standard passive objects and process objects, all public functions have to be virtual, otherwise, the non-virtual function calls will not be transformed into IPC. This drawback can be alleviated with C++ compilers providing "all-virtual" option; here there is a choice between paying the price of all virtual functions, and reusability. Of course, this feature requires recompiling all files involved, but is probably a small price compared to the reuse that can be obtained.

\section{Control programming}

So far, we have only examined and defined the features of $\mathrm{C}++/ /$ which deal with the global aspects of the programming model, such as the nature of processes and their interactions. This section describes how the control flow of processes is specified, i.e. how behavior, communication, and synchronization of active objects are programmed.

\subsection{Centralized and explicit control}

Control can be decentralized, that is, distributed throughout a program or, alternatively, control can be centralized, i.e. gathered into one place in the definition of a process, independently of the function code.

Decentralized control makes reuse of function code difficult for two reasons. First, functions designed in a sequential framework cannot be reused in a parallel one just as they are, as elements of control must be added 
to them. Second, when a new process class is obtained through inheritance, the new class often needs to change the synchronization scheme used in the original class. If control is embedded in function bodies, this may not be feasible. This leads to the following choice:

$\diamond$ Model: processes have a centralized control.

It allows function reuse for both sequential and process classes without changes to the bodies of such classes.

Program 1.2 presents partial code for the library class Process. After creation and initialization, a process object executes its Live routine. This routine describes the sequence of actions which that process executes during its lifetime. The process terminates when the Live routine completes.

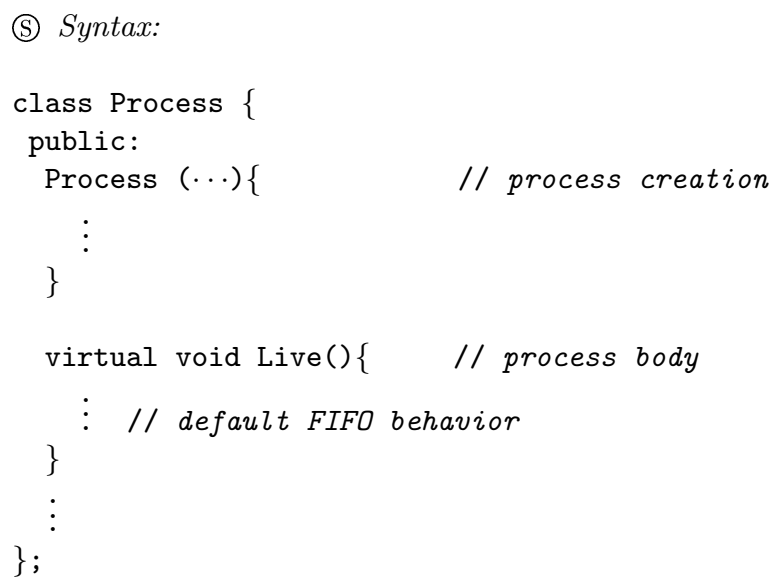

Program 1.2. The Process class.

Another design decision that must be made in concurrent object oriented systems is whether process control is implicit or explicit. Control is explicit if its definition consists of an explicitly programmed thread of control. Otherwise, control is implicit, which in practice usually means that it is declarative.

Our argument is that (see [16] for a complete discussion):

1. sometimes programmers need explicit control;

2. implicit control permits reuse of synchronization;

3. no universal implicit control abstraction exists; and

4. explicit control allows us to build implicit control abstractions.

As a consequence, the basic mechanism for programming process behaviors in $\mathrm{C}++/ /$ is:

\section{$\diamond$ Model: explicit control.}

Explicit control programming consists of defining the Live routine of the Process class and its heirs (Program 1.2) using the sequential control structures of $\mathrm{C}++$. All of the expressive power of $\mathrm{C}++$ is available, without any limitation. For instance, the process body of a bounded buffer can be defined as in Program 1.4.

Besides explicit control, other features are needed in order to construct abstractions for concurrent programming. These features permit $\mathrm{C}++/ /$ to explicitly service requests. First, defining a process's thread of control often consists of defining the synchronization of its public member functions. Since such an activity requires dynamic manipulation of $\mathrm{C}++$ functions, we need:

$\diamond$ Model: member functions as first class objects.

In practice, only some limited features, such as the ability to use routines as parameters, and system-wide valid function identifiers, are needed. 
To fill that need, we provide the function $\operatorname{mid}()$ to return function identifiers. Its usage is:

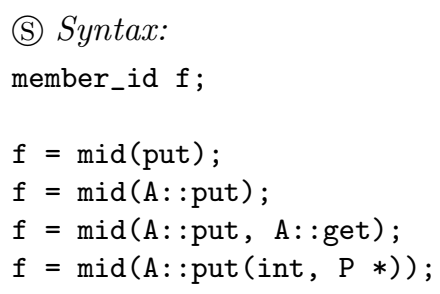

In order to deal with overloading, this function returns either a single identifier, or a representation of all adequate functions.

In the same way, because we need to explicitly program request servicing, we must be able to manipulate requests as objects (i.e., to pass them as parameters of other functions, to assign them to variables, and so on). We require:

$\diamond$ Model: requests as first class objects.

In $\mathrm{C}++/ /$, a particular class ( Request ) models the requests; every request is an instance of this class.

Finally, to be able to fully control request servicing, programmers must have

$\diamond$ Model: access to the list of pending requests.

This is given through the Process class, with a specific member named request_list that contains the list.

With these three facilities in place, it is possible to program the control of processes in diverse and flexible ways.

\subsection{Library of service routines}

Service primitives are needed to allow programmers to program control explicitly. Usually, programmers are given only a few such primitives, mainly because they are made part of the language itself as syntactical constructions (e.g., the serve instruction of Ada). With the primitives we define, it is possible to program a complete library of service routines [13]. Some of these are shown in Program 1.3, where $f$ and $g$ are member identifiers obtained from the function mid() introduced in the previous section.

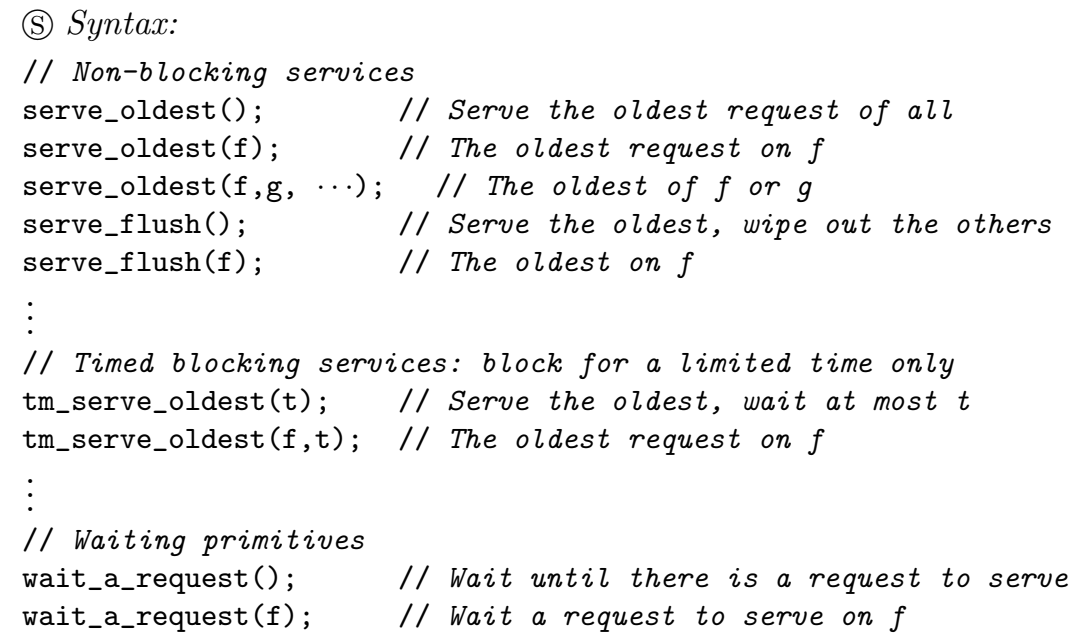

Program 1.3. A library of service routines.

These functions are defined in the class Process, and can be used when programming the Live routine. There is no limitation in the range of facilities that can be encapsulated in service routines. Timed services 
are an example of such expressiveness; selection based on the request parameters is another. Moreover, if a programmer does not find the particular selection function he needs, he is able to program it. Thus, libraries of service routines specific to particular programmers or application domains can be defined.

Another important point concerns efficiency: concurrent policies are determined within the context of each process, based on local information rather than by using IPC, avoiding problems like polling bias [27]. This is an important advantage in distributed programming.

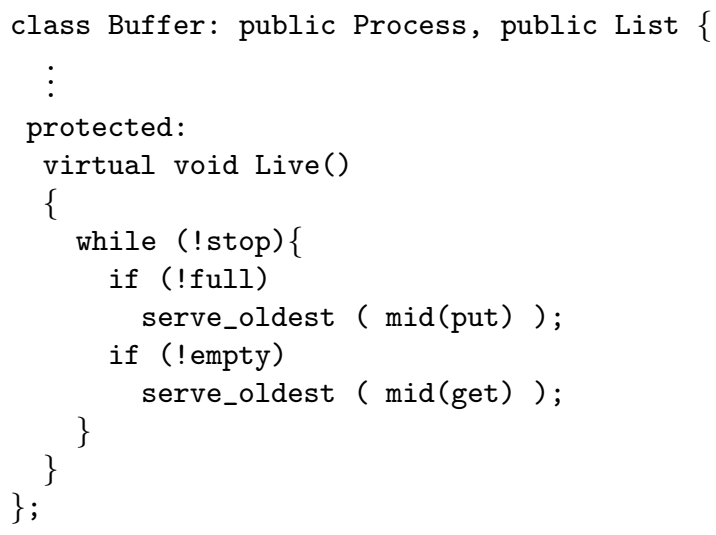

Program 1.4. An explicit bounded buffer example.

As an illustration of the use of explicit control programming, Program 1.4 presents a C++// implementation of a bounded buffer. This definition implements a specific policy: when the buffer is neither full or empty, the buffer alternates service on put and get. This policy is clearly not the only possible one.

This is an example of explicitly fine-tuning the synchronization of processes. While this might be very important in some contexts, we might want to program within a more abstract framework in others, ignoring the implementation details, through the definition of libraries of abstractions [16].

As we finished the control programming of processes, Figure 4 summarizes the basic features on the $\mathrm{C}++/ /$ model.

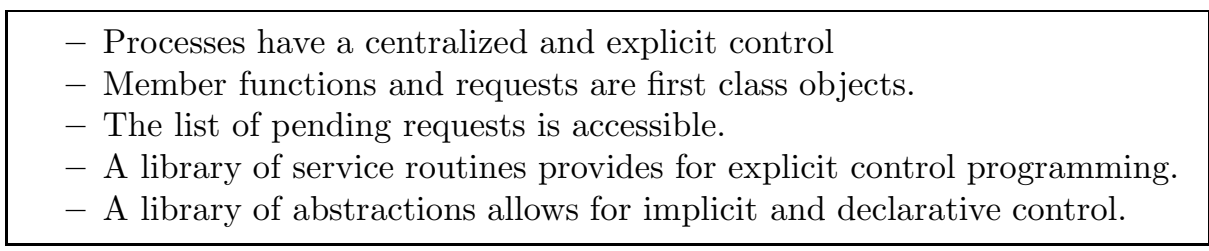

Figure 4. Control programming in $\mathrm{C}++/ /$.

\section{Programmation, Environment and implementation}

\subsection{A programming method}

Because it is rather difficult to evaluate performances of distributed system before they actually run, we believe that definition of processes has to be postponed as much as possible, and should be flexible and adaptable. The programming guide we develop in this section applies this principle, made possible by the features of the $\mathrm{C}++$ // model. 
The first step is a standard, sequential, object-oriented design, possibly including object identification, interface and topology design, and sequential implementation $[9,35]$. The next steps deal with parallel design and are specific to the language and technique we developed.

Processes are a subset of classes. Because object-oriented design usually gives a finer-grained decomposition than structured design or information hiding methods (because every type is a module, and every module is a type), there is no need for re-structuring. The classes remaining passive are commonly used without any changes.

Here is an example of how an entity a declared:

A* a;

get assigned with a process object of type P_A (heir of A):

$\mathrm{a}=$ new P_A $(\ldots)$;

A function call on a is now executed on an asynchronous basis (the caller does not wait for its completion). This automatic transformation of synchronous call into asynchronous one is crucial to avoid routine redefinition. An inherited routine may use the result of a function call issued to the process:

res $=a->f c t($ parameters $)$;

:

res->g( parameters);

In this case, the wait-by-necessity handles the situation. Without this automatic data-driven synchronization one would have to redefine the current routine in order to add explicit synchronization.

These model properties ensure that most of the inherited routines remain valid for the process class. However, some special cases need re-programming.

Figure 5 presents the significant steps of the method.

1. Sequential design and programming.

2. Process identification.

3. Process programming:

- Define each process class (Process or abstraction class).

- Define the activity (Live).

- Use the process classes with polymorphism.

4. Adaptation to constraints:

- Refine the topology.

- Define new Processes.

Figure 5. The 4 steps of the method.

\subsection{Environment}

This section briefly describes the facilities supporting the development of $\mathrm{C}++/ /$ programs, including the compilation of source code, executable generation (see Fig. 6), and more specifically a mechanism for mapping active objects onto machines.

Mapping assigns each active object created during the execution of a $\mathrm{C}++/ /$ program to an operating system process on an actual machine or processor. In order to avoid confusion, we call the sub-system consisting of one active and all its passive objects a language process, and use the term OS process for the usual notion of an operating system process. 


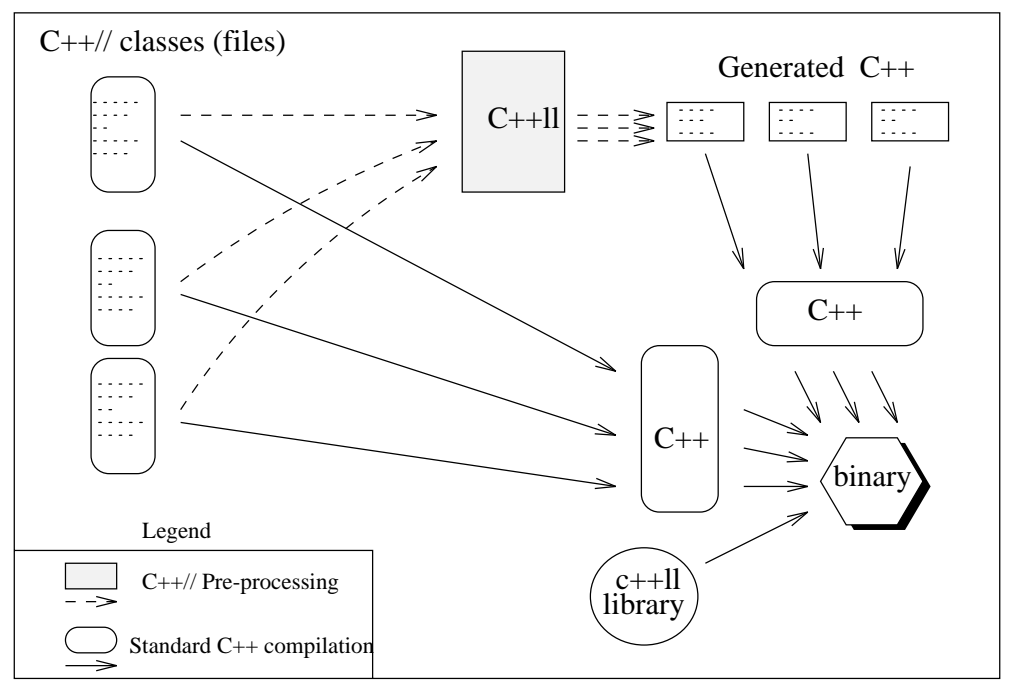

Figure 6. Compilation of a $\mathrm{C}++/ /$ system.

The mapping of a language process to an OS process on a particular processor is controlled by the programmer through the association of two criteria:

\section{$\diamond$ Model:}

(1) the machine where the language process is to be created;

(2) the light-weight or heavy-weight nature of the language process.

The machine itself can be specified in two ways. The first method is to specify a virtual machine name, which is simply a string. This name is related to an actual machine name by a translation file called . c++ll-mapping. The $\mathrm{C}++$ // system looks for this file first in the directory in which the process is running, and, if it is not found there, in the user's home directory. An example of such file is:

\section{$\square$ File:}

FILE .c++ll-mapping

$\begin{array}{cl}\text { // virtual name } & \text { actual name } \\ \text { Server } & \text { Inria.Sophia.fr } \\ \text { S1 } & \text { wilpena.unice.fr } \\ \text { S2 } & \text { 192.134.39.96 } \\ \text { S3 } & \text { // current machine } \\ \text { P1 } & \text { I3S-1 } \\ \vdots & \vdots \\ \text { P6 } & \text { INRIA-1 }\end{array}$

The other technique used to specify a machine is to use a language process that already exists. In this case, the new process is created on the machine where that language process is running. With this technique, processes can be linked together to ensure locality.

The light-weight switch permits creation of several language processes inside a single OS process. In the heavy-weight case, only one language process is mapped to each OS process. The user accesses these switches 


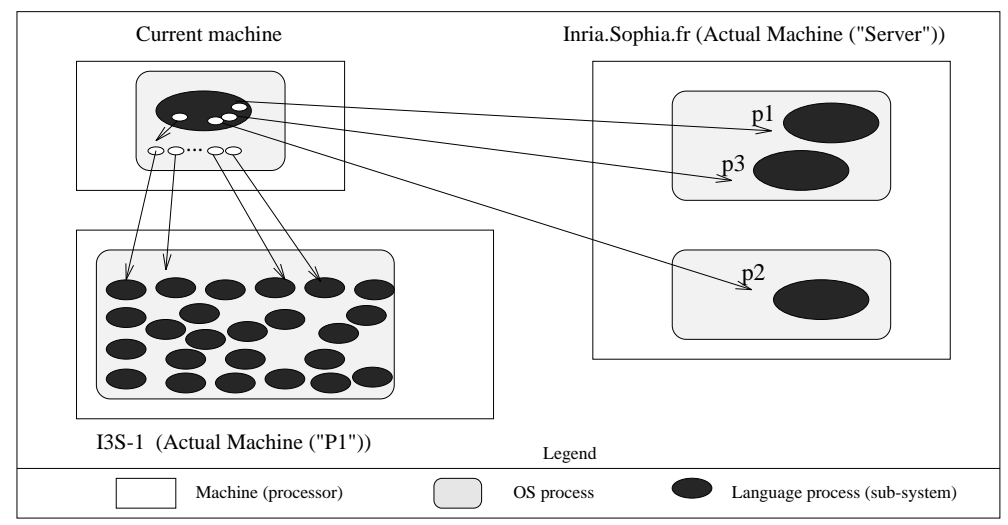

FiguRE 7. Example of mapping.

through a class called Mapping:

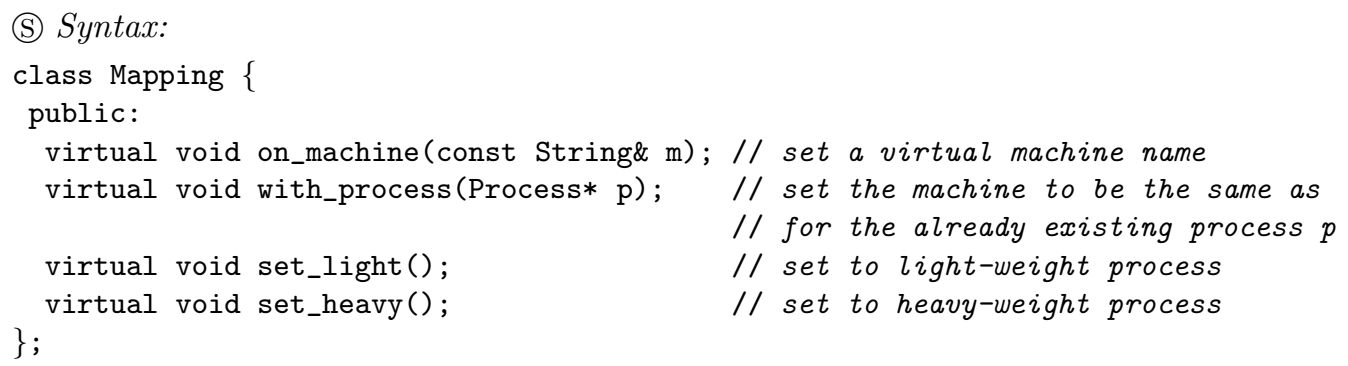

When a program creates a language process, an object of type Mapping can be passed to new in order to specify the desired mapping of the new process. Program 1.5 presents the syntax used for this. With the .c++ll-mapping file taken from above, Program 1.5 produces part of the mapping presented in Figure 7.

$\nabla$ Example:

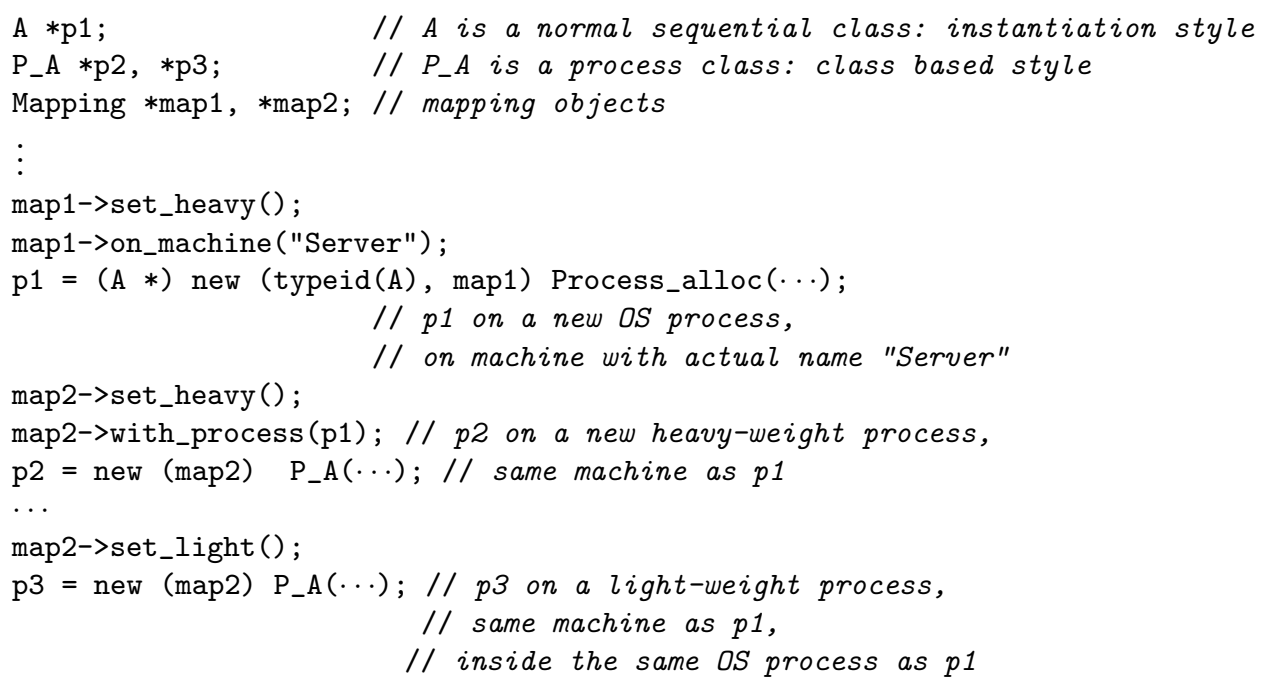

Program 1.5. Mapping processes to machines. 


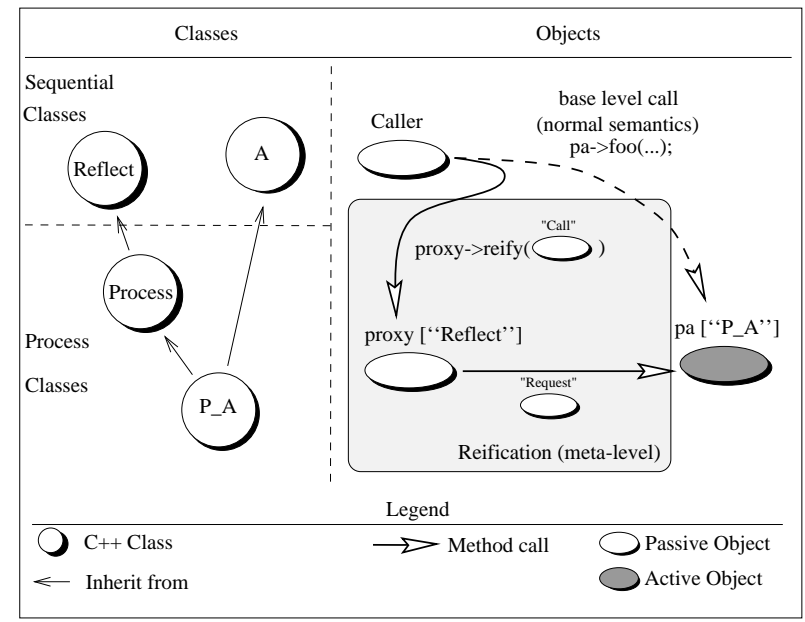

FiguRE 8. Reification of calls.

\subsection{Implementation}

This presentation goes beyond implementation details since the technique we use - reification-also provides for customization and extension of our system.

\subsubsection{A reflection-based system}

The $\mathrm{C}++/ /$ system is based on a Meta-Object Protocol (MOP). There are various MOPs [32], for different languages and systems, with various goals, compilation and run-time costs, and various levels of expressiveness. MOP techniques have been used in many contexts in particular for parallel and distributed programming [11, 20,34 .

Within our context, we use a reflection mechanism based on reification. Reification is simply the action of transforming a call issued to an object into an object itself; we say that the call is "reified". From this transformation, the call can be manipulated as a first class entity, i.e. stored in a data structure, passed as parameter, sent to another process, etc.

A meta-object (Fig. 8) captures each call directed towards a normal base-level object; a meta-object is an instance of a meta-class. In some ways, a proxy, a local object that permits to access a remote one [8,22,39], is a kind of meta-object.

\subsubsection{A MOP for $\mathrm{C}++$ : basic classes}

The main principle of our MOP for $\mathrm{C}++$ is embodied in a special class, called Reflect, which presents the following behavior:

$\diamond$ Model: all classes inheriting publicly from Reflect, either directly or indirectly, are called reified classes, a reified class has reified instances; all calls issued to a reified object are reified.

This last requirement is important for reusability, as it permits users to take a normal class, and then globally modify its behavior, to transform it into a process.

Figure 8 illustrates reification. The creation of an instance of a Reflect class returns a meta-object (a proxy) for the type being passed in as the allocator's first parameter. From this mechanism, we implement the basic classes of our programming model described in Section 2.1.

\subsubsection{Customization and extension of $\mathrm{C}++/ /$}

The MOP we just presented is independent of any parallel programming model. The classes of the MIMD model (such as Process) we described in this paper are programmed on top of the MOP, without any compiler 
modification. An important consequence of this is that other parallel programming models, such as sharedmemory MIMD or SPMD, can be defined on top of the MOP. The wait-by-necessity implementation, for instance, is achieved through a class Future which uses reification by inheriting from Reflect. Such an open system, or open implementation [32], is extensible by the end-user or by developers of new libraries, and adaptable to various needs and situations, such as the ones presented in the two following sections.

Notice that this MOP has been adopted as the level 0 of a standard framework for parallel $\mathrm{C}++$ systems, designed in the context of the EUROPA Parallel C++ working group, formerly funded by the EU [17]. In this framework, other $\mathrm{C}++$ libraries for parallel computing, such as $\mathrm{UC}++$ (featuring constructs which are variations of those found in $\mathrm{C}++/ /$, like active objects, asynchronous communications and futures) were implemented on top of this MOP. A similar effort in the United States is $\mathrm{HPC}++$, and now, OpenHPC ++ [24], which supports constructs to develop both task as well as data parallel applications in $\mathrm{C}++$. [26] explains how it would be possible to implement the parallel STL model of HPC++ on top of the MOP adopted within the EUROPA working group.

They are some other few extensions of $\mathrm{C}++$ for parallel computing [41] that are built upon reification mechanisms, in particular, onto the meta-object protocols OpenC $++[20]$ and $\mathrm{MPC}++[31]$.

\section{Sharing PASSive OBJECtS AMONG ACTIVE OBJECTS}

This section presents a mechanism for sharing objects [19] when two active objects that reside in the same address space (in the same process) want to access the same passive object in read mode.

\subsection{The SharedOnRead framework}

A crucial point of the standard $\mathrm{C}++/ /$ model, is where active objects are created. A $\mathrm{C}++/ /$ programmer has several choices to determine the machine (or node) where a new active object will be created: (1) give the machine name, (2) provide an existing active object in order to use the same machine. But central to the issue is that, in both cases, the programmer has two options to create the new active object: (a) in an existing address space, (b) in a new address space. In case (a), several active objects will be able to share the same address space - threads belonging to a same heavy-weight process are used to implement active objects. Let us note that even when active objects in the same address space communicate, passive objects are still transmitted by copy. This potentially time and space consuming strategy is mandatory if we want the program semantics to be constant, whatever the mapping is. However, in some cases, sharing is actually possible, and copying large objects could be avoided. The SharedOnRead mechanism was defined to make possible such optimization, and to provide a general strategy that keeps the semantics unchanged when the mapping varies.

\subsubsection{Strategy}

Upon a communication between two subsystems that are within the same address space, instead of copying a parameter if it is of type SharedOnRead, we just share it; otherwise, there is no alteration of the copy semantics if the two subsystems are not in the same address space (cf. Fig. 9). The SharedOnRead is dearly related to the copy-on-write mechanism that can be found in operating system (Mach [37] or Orca [6,29] are using it). However, the strategy is slightly different in copy-on-write techniques: one wants to copy only when it is necessary, instead, with the SharedOnRead mechanism, one wants to share data on read operations whenever it is possible (i.e. same address space).

While this idea is quite simple, a mechanism is needed in order to maintain the copy semantics of $\mathrm{C}++/ /$ that should apply everywhere, even when the two subsystems are mapped in the same address space. We can notice that objects will be able to be shared by several subsystems only as long as they are not modified by one of the subsystems. In order to be accurate, the strategy needs to make a distinction between read and write accesses, and also needs to know when a subsystem forgets a SharedOnRead object (the subsystem suppresses its reference to this object). 


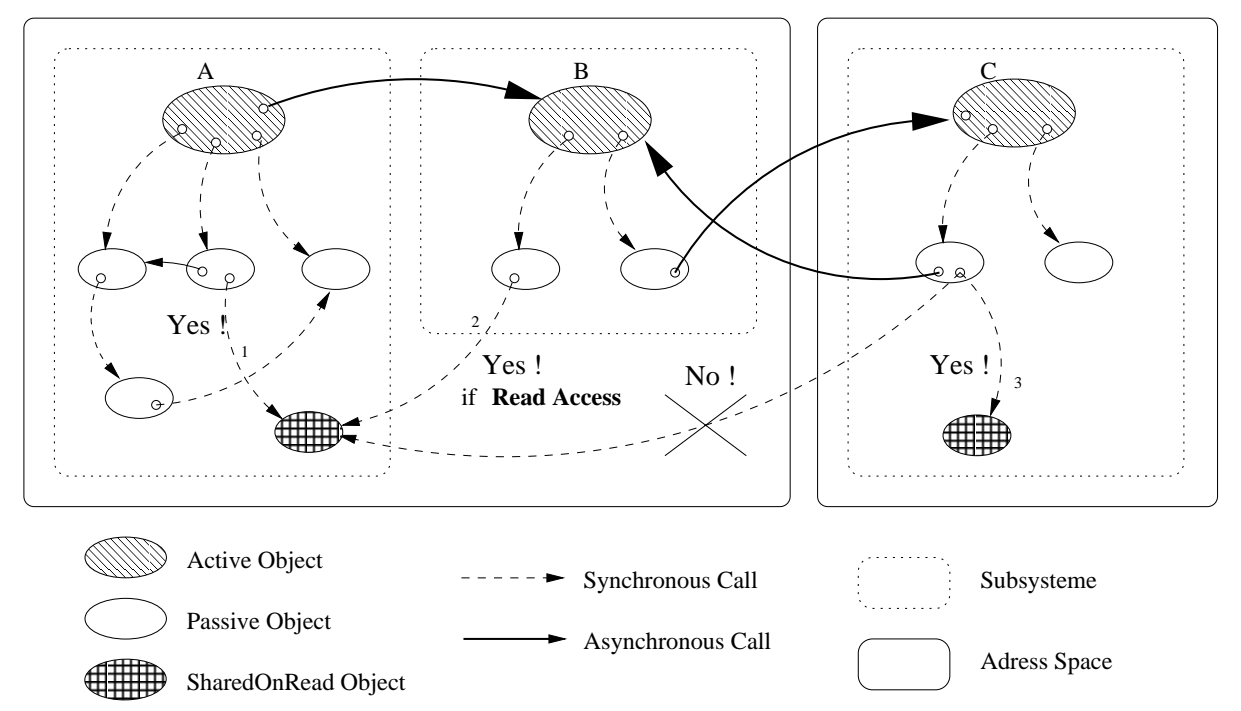

FIGURE 9. SharedOnRead objects within several address spaces.

The requirements at the design level are the following:

$\diamond$ Model:

(1) When a SharedOnRead object is used as a communication parameter between two subsystems being in the same address space, the original object is not copied, but instead a new reference is memorized (a counter is incremented within that object).

(2) A read access is freely achieved (from both the owner's subsystem or another one).

(3) Upon a write access (from both the owner's subsystem or another one), if the counter value is more than 1 , a copy of the object is made. The modification applies to the copy. The counter of the previously existing object is decremented; the counter of the copy is set to 1 .

(4) Upon a forget operation, the counter is just decremented. When reaching zero, the object is automatically garbaged.

\subsubsection{Programmer interface and implementation}

As a design decision, we choose to give users the control over which objects should be SharedOnRead and which should have the standard systematic copy behavior.

$\diamond$ Model: A SharedOnRead object is an instance of a class that inherits directly or indirectly from the $\mathrm{C}++$ // SharedOnRead class.

The SharedOnRead class is:

(S) Syntax:

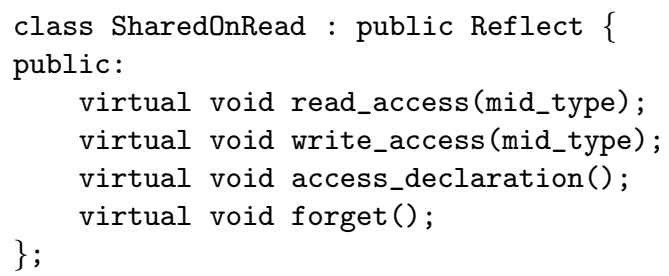

and as such provides several member functions whose usage is now described. read_access and write_access, are both used to specify how the data members are accessed by a given method. If read_acess is selected by 
the programmer for a given method, the programmer declares that, for this function, data members are never changed, alas if write_access is selected, data members can be changed. These two functions take a mid_type parameter which is unique for all the member functions in the program; the mid function (see Sect. 3.2) provides that unique identifier for a function name. The access_declaration function has to be redefined in order to specify for each public member function its read or write nature: write access is the default behavior. A SharedOnRead user has to take care of that and it is his responsibility to check for each method in the class which ones are leaving the object in the same state and which ones are making modification to the object state. Lastly, forget must be called so as to declare that this SharedOnRead object is not used anymore. This have to be dealt with explicitly because SharedOnRead objects cannot be aware that they are not referenced anymore.

Program 1.6 gives an example of the use of the SharedOnRead mechanism, in which the programmer has just to define a new class that inherits from an existing class and the SharedOnRead one, the only additional programming work being to redefine the access_declaration function.

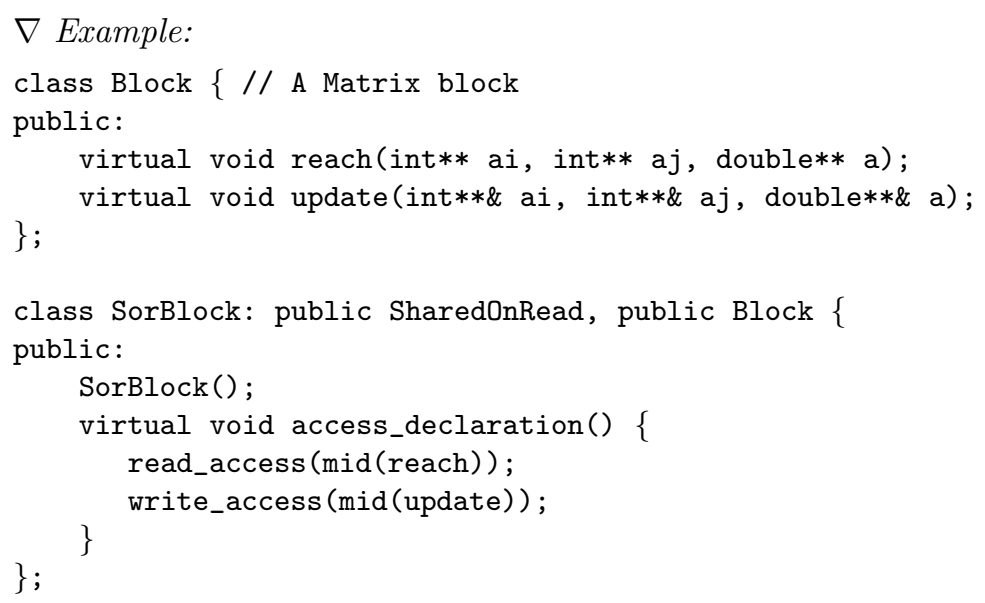

Program 1.6. Programming a SharedOnRead block in a matrix-based example.

The implementation of the SharedOnRead class is based on the reification mechanism provided by $\mathrm{C}++/ /$. Inheriting from the class Reflect, all the SharedOnRead member functions are reified: the functions are not directly executed but are derouted in a specific proxy where all the necessary implementation is defined and achieved (update of the counter, copy when necessary, etc.). After this step, the proxy executes the function.

\subsection{Benchmark application}

\subsubsection{Parallel linear algebra}

We have tested the SharedOnRead mechanism on basic linear algebra operations commonly used in iterative methods [38]. The key point for efficient parallel implementation of iterative methods are good performance of the distributed sparse matrix/vector product, and distributed dot product since these operations are at the heart of all basic Krylov algorithms [36]. In this context it is crucial to avoid unnecessary copies in matrix operations.

As defined in [36] we may focus on a reduced set of operations:

- dense SAXPY, $Y:=\alpha X+\beta Y$ with $Y, X \in \mathbb{R}^{n \times p}, \alpha, \beta \in \mathbb{R}$

- dense or sparse matrix product $Y:=\alpha A \cdot X+\beta Y$ with $Y \in \mathbb{R}^{m \times p}, A \in \mathbb{R}^{m \times n}, X \in \mathbb{R}^{n \times p}, \alpha, \beta \in \mathbb{R}$.

Parallel numerical linear algebra is concerned with data distribution of the matrix arguments in the above operations. In our case we will only consider a block distribution scheme of matrices which is widely used [21] and well suited for these applications. With these kind of distributions, we have to do dense matrix saxpy operations and dense or sparse matrix products. 
These operations are implemented as a method of a class Matrix representing a block partitioned matrix. The methods are:

- Matrix: :scal(double alpha, Matrix* B, double beta)

This method performs the matrix saxpy operation this $=\beta \cdot$ this $+\alpha \cdot B$.

- Matrix: :axpy(double alpha,Matrix* A,Matrix* X,int $\mathrm{mm}$ )

This method performs the matrix operation this $=\alpha \cdot A \times X+$ this, and this $=\alpha \cdot A \times X$ if $\mathrm{mm}==1$.

Here, the distributed objects are the blocks of the matrix which may be called CSC since they are Compressed Sparse Column (potentially sparse) matrices.

\subsubsection{From sequential to parallel matrices in $\mathrm{C}++/ /$}

A sequential matrix contains a list of CSC objects, each of these objects holding a Block. This Block is responsible for allocating all the arrays representing the matrix. If we want to parallelize these classes using $\mathrm{C}++/ /$, we only have to redefine the Matrix constructors. These constructors create the distributed CSC objects of type Block or SorBlock (see Program 1.6) depending if we want to use the SharedOnRead mechanism or not. A distributed CSC object (CSC_ll object) can be created just by inheriting from CSC and the C++// class Process. All the functions presented above (scal, axpy, ...) come unchanged from the sequential classes.

Program 1.7 presents the sequential version for a dense axpy function, which will be reused unchanged in the $\mathrm{C}++/ /$ version. Thanks to polymorphism compatibility, the two A and X variables can be CSC_ll objects. If the SharedOnRead is used in the matrices construction, the block() function returns a SorBlock object: as such, as bl1 and bl2 must be accessed only in read mode, we will use them directly if they are located in the same address space, without generating any copy. On the contrary, the mine variable, which represents the local Block of the CSC object, will be modified, so update has been declared in write mode in Program 1.6.

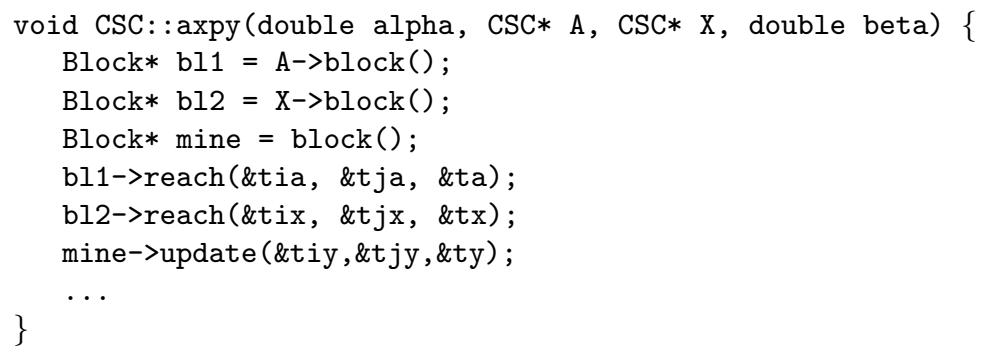

Program 1.7. Sequential dense CSC_Block product.

\subsubsection{The MPI version}

Our objective is to apply the SharedOnRead mechanism and prove that this yield to performances as good as the ones obtained with MPI, but with more transparency and flexibity for the programmer due to the objectoriented model of programming. The MPI [2] implementation requires to redefine all the functions in order to take into account the fact that not the same operations must be executed depending on the processor they are executing on: MPI is very intrusive. This means that the programmer has to add a lot of MPI calls in the original sequential code in order to derive the parallel version. Moreover, this makes it difficult to the programmer to go back and forth from the sequential to the parallel version. Furthermore, MPI is a message passing library thus parallelism is explicit, and the programmer has to directly deal with distribution and communication.

\subsubsection{Performances}

The following tests were performed on a network of 4 Solaris Ultra 1 with $128 \mathrm{MB}$ of memory and a $10 \mathrm{Mb} / \mathrm{s}$ Ethernet link. The MPI tests use the LAM library (http://www.mpi.nd.edu/lam/).

Since the runtime is not based on a virtual shared memory, the standard distribution algorithm can imply that several active objects (representing CSCs holding matrix blocks) get mapped within the same address space on each workstation, while others get mapped within different address spaces. Recall that the SharedOnRead 


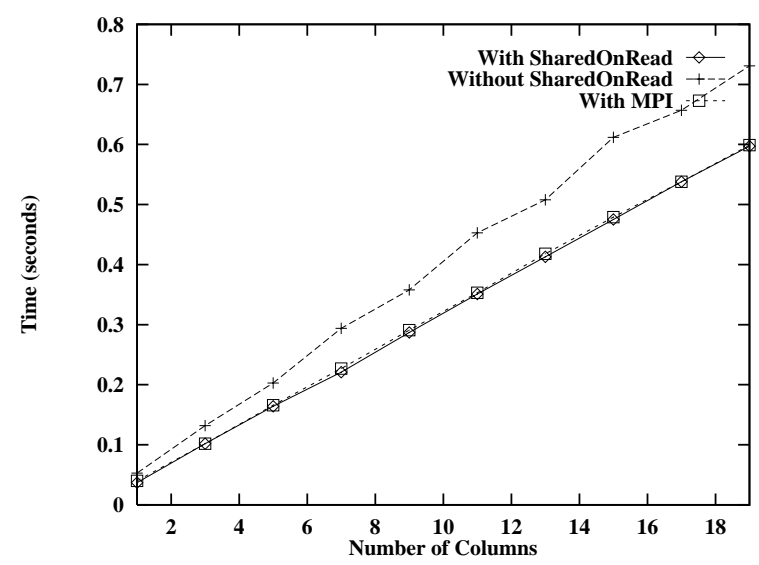

FiguRE 10. SAXPY with 4 computers.

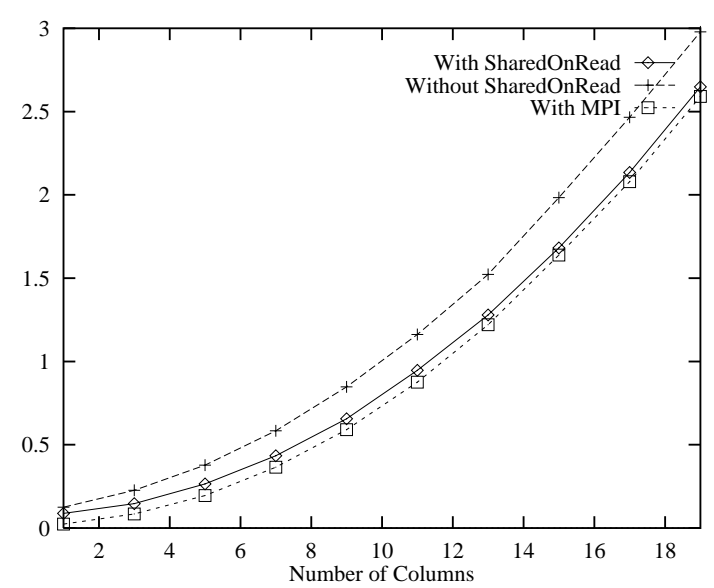

(a)

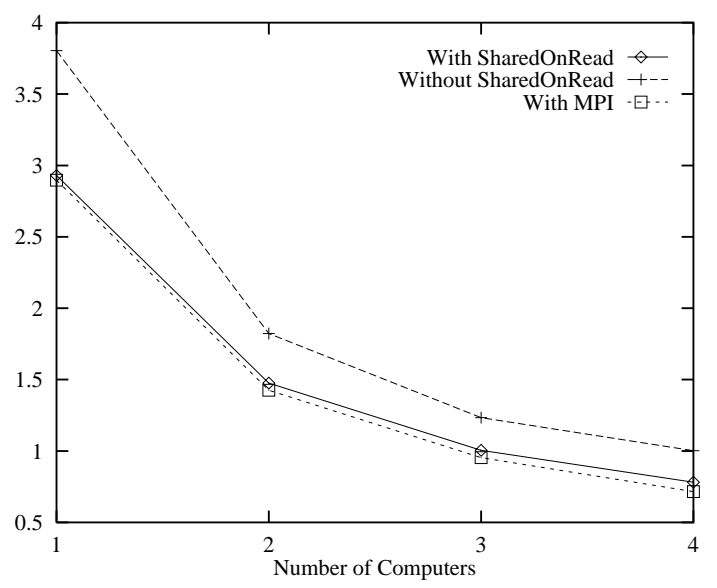

(b)

Figure 11. Dense matrix product (a) duration in seconds using 4 computers (b) speed-up.

optimization applies when a computation occurs within the same address space. As demonstrated below this is sufficient to achieve consequent speedup. If we were on an SMP architecture, then the benefits would be even greater since there would be opportunity for sharing all the matrix blocks.

Figure 10 presents the performances for a scal (cf. Sect 5.2.1) calculation. Matrices used during these tests were rectangular matrices with 90449 rows and a variable number of columns. The use of SharedOnRead objects demonstrates a speed-up between 20 and $25 \%$ compared to the non optimized $\mathrm{C}++/ /$ version. When compared with the MPI version, we cannot distinguish any difference between $\mathrm{C}++/ /$ with SharedOnRead and MPI. One important point to notice is the fact that the non optimized C++// version (without SharedOnRead objects) presents more and more overhead when the matrix size increases. The main reason is that this version requires many communications between the different active objects even if they are located in the same address space. In the SharedOnRead version, the two blocks represented by two active objects are mapped within the same process, so communications and copies are avoided.

Figure 11a presents performance results for a dense matrix product. Again, the performances of the experiment using the non optimized $\mathrm{C}++/ /$ version is between 20 and $30 \%$ slower than the MPI one. Between the 


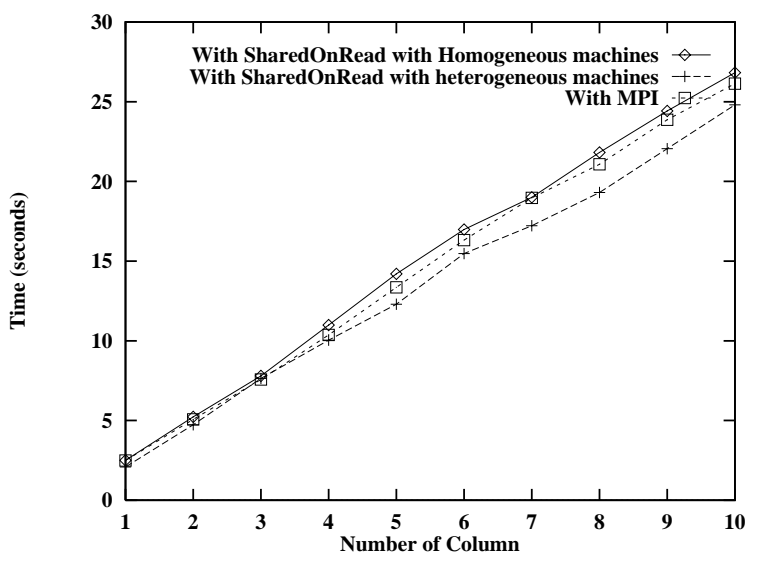

FiguRE 12. Sparse matrix product with 4 computers.

$\mathrm{C}++/ /$ version and the MPI one, the overhead is constant. But the SharedOnRead version and the MPI one do not behave exactly the same: the $\mathrm{C}++/ /$ solution sums the local matrix in a sequential way because it reuses the sequential code, whereas the MPI version requires communication during the reduction step. Figure 11b presents the speed-up obtained with 4 computers for the 3 different experiments. All calculations on dense matrices are perfectly scalable; with 4 computers, the speed-up is around 3.9. We can observe that the overhead of the non optimized $\mathrm{C}++/ /$ version is constant whatever the number of computers we use.

At last, Figure 12 presents performance results for a sparse matrix product. A first point to notice in such a case is that the add function of the matrix had to be rewritten in the $\mathrm{C}++/ /$ version: the reduction being critical in this benchmark, it was important to compute it in parallel. The second important aspect of this benchmark deals with the platform architecture. All the previous tests were made on homogeneous computers: the same CPU, at the same frequency, with the same amount of memory. This last test was performed on an heterogeneous platform. Two of the four Ultra 1 were replaced with two Ultra 2 with 192 MB of memory. Within this new architecture, the MPI program has the same performance as in the homogeneous test. In the $\mathrm{C}++/ /$ case, the benchmark demonstrates that the object-oriented version is actually more efficient than the MPI one. While MPI is subject to synchronization barriers, the $\mathrm{C}++/ /$ version automatically takes advantage of the asynchronous model. In that case, the reduction of the two local matrices of the fastest computers can start even if the computation on the slowest computers has not finished.

\section{Overlapping COMmunication With COMPUtation}

This section presents the concepts and an implementation of an overlapping mechanism between communication and computation [7]. This mechanism allows to decrease the execution time of a remote method invocation, especially in the context of important transfers, such as matrices.

A general idea to lower communication costs is to overlap communication with computation, thus yielding to a pipeline effect regarding messages transmission. Any attempt to exploit this opportunity needs to rely on non-blocking elementary communications, such as for instance, asynchronous send and receive primitives as provided by well-known message-passing libraries (e.g. PVM [1] or MPI [2]).

For code readability and portability purposes, one additional requirement is to make the use of the overlapping technique as much transparent as possible for programmers. As such, we reject distributed hand programmed solutions where the programmer would himself split the data to be sent into smaller pieces, asynchronously send each piece in turn thus "feeding" the pipeline, while at the receiver side, explicitly and repetitively receive each new piece and goes on with it in the related computation. 

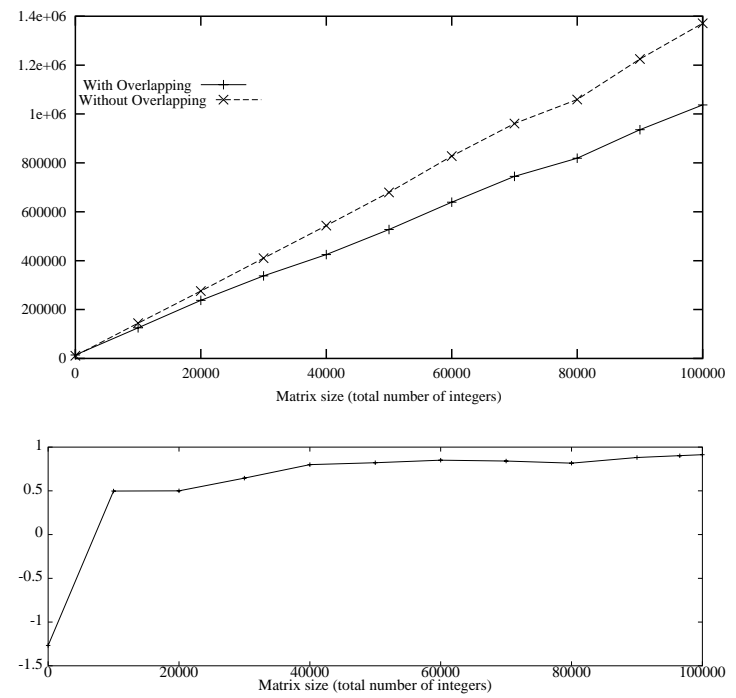

FIGURE 13. Execution of the remote service (caller side, total_duration in $\mu$ s) and corresponding benefit $(G)$ obtained from using the overlapping technique on a LAN.

important point is that the order the various parameters are first used should closely follow the order they are sent and received. So, the position of later parameters in method signatures becomes important.

Implementing the overlapping technique requires only minor modifications in the language runtime support. At the MOP level, the main modification is to write a new generic function to flatten requests: this function builds a first fragment which holds the request header and the non-later parameters, and then one fragment for each parameter of later type. Then at the runtime level, the first fragment is sent and its service will consist to create a $\mathrm{C}++/ /$ future type for each missing part of the request, i.e., for each later part of the request parameters. Concerning the remaining fragments, they will be subsequently sent and served as follows: transparently update the corresponding awaited request parameters, i.e. the corresponding future objects.

\subsection{Benchmark}

\subsubsection{Description of the experiment}

We designed a simple test and benchmarked it. This test must not be considered as a real application, but as a means to validate the effectiveness of the technique. It is based on the remote call of the method OpMatrix::rang(...) (see Program 1.8) which takes two matrices, squares the first one, and adds the second one. As the second matrix $\mathrm{m} 2$ is of type Matrix_later, it can be used as a parameter of OpMatrix::rang(...). The remote service can start as soon as the request id and the non-later parameters have been received. Experiments not using the overlapping technique are easily conducted: define $\mathrm{m} 2$ as an instance of Matrix instead of Matrix_Later.

The technique should allow to overlap the transmission and reception of the later parameter (i.e. the matrix $\mathrm{m} 2$ ) that is only useful for the second part of the service execution (i.e. $\mathrm{m} 2 \rightarrow \mathrm{plus}(\mathrm{m} 1)$ ) with the remote execution requiring only $\mathrm{m} 1$ (i.e. the method $\mathrm{m} 1 \rightarrow$ square()). Compared with an execution not using the overlapping technique, the duration of $\mathrm{m} 1 \rightarrow$ square () (noted $d 1$ in the following) should increase, since, at the same time, the remote processor has also to manage the reception and update of the matrix $\mathrm{m} 2$.

\subsubsection{Results}

Two Sun Solaris 2.6 workstations with $128 \mathrm{MB}$ of RAM, interconnected by a $10 \mathrm{Mb} / \mathrm{s}$ Ethernet are used. The curves plotted in Figure 13 show a decrease of the dom $\rightarrow$ rang $(\mathrm{m} 1, \mathrm{~m} 2)$ execution time, and an almost optimal gain as computed by $G$. 

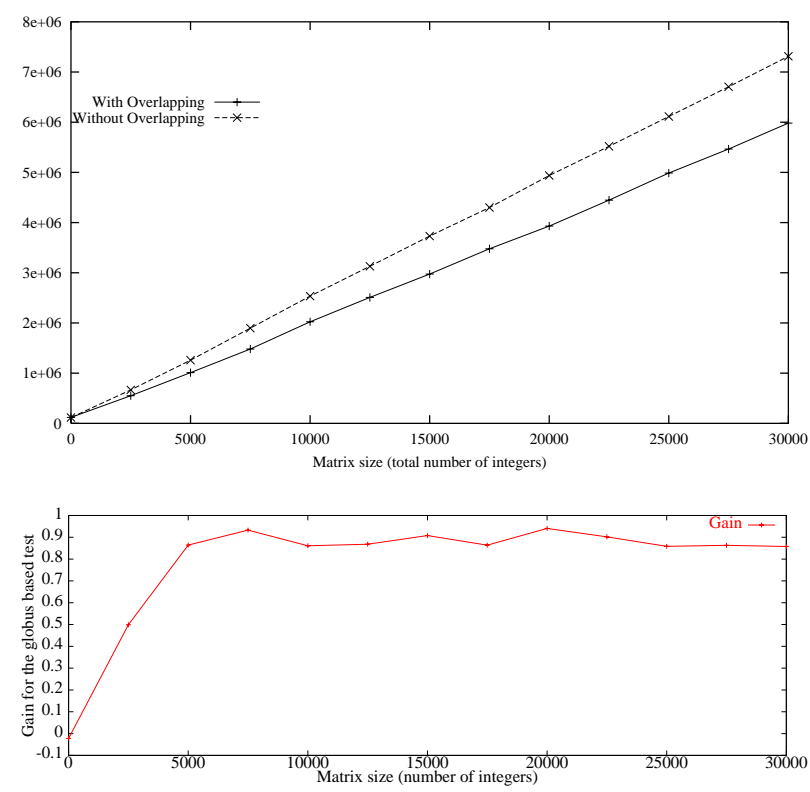

FIGURE 14. Execution of the remote service (caller side, total_duration in $\mu$ s) and corresponding gain. This corresponds to one Globus-based test between USA and France during night period, with (d1) 300 times longer than in Figure 13.

Let us define the gain $(G)$ in order to give a concrete estimation of the benefit.

$$
G=\frac{\text { duration }_{\text {not_using_overlap }}-\text { duration }_{\text {using_overlap }}}{\text { later_parameters_transfer_duration }} .
$$

The duration for transferring later parameters, i.e. $\mathrm{m} 2$, is estimated by sending a $\mathrm{C}++/ /$ object of the same size, not counting the - small - additional cost that would be required for managing a later parameter (a few milliseconds).

Scalability. The overlapping technique used in this context where lightweight processes are available, scales very well. Moreover, we deduce against our past experiences that only runtime supports using lightweight processes can scale so well. Indeed, benchmarks conducted in the context of $\mathrm{C}++/ /$ on top of PVM proved that the amount of data that could be sent and received while the remote service is in progress, is bounded by the remote receiving buffer size. The fundamental reason is that the transport-level layer can not gain the receiver process attention while this latter is engaged in a remote computation (i.e. $\mathrm{m} 1 \rightarrow$ square()), due to the lack of a dedicated concurrent receiving thread.

WAN-based results. On WAN-based environments (see Fig. 14), sparing the transmission time of even a few bytes $^{1}$ yields a gain that the overhead of the technique can not override (very small compared to the high transmission delays). But, one should notice that the duration of the remote computation is of course an other crucial point. Indeed, if it is really too short compared with the transmission speed, almost no communication overlap occurs. This is why the Globus-based [25] grid experiment plotted in Figure 14 assigned $d 1$ to be 300 times higher than in experiments plotted in Figure 13. In concrete situations, such a high-computation duration is not an unrealistic experimental assumption, as transmitting a large or even huge volume of data to remote computers (especially on a grid) is justified by the need to execute quite costly computations on these data.

\footnotetext{
${ }^{1}$ More precisely, the total duration for the test in Figure 14 using matrices $m 1$ and $m 2$ of 2500 integers decreases from $680816 \mu$ s not using the overlapping technique to $556446 \mu$ s when using it.
} 


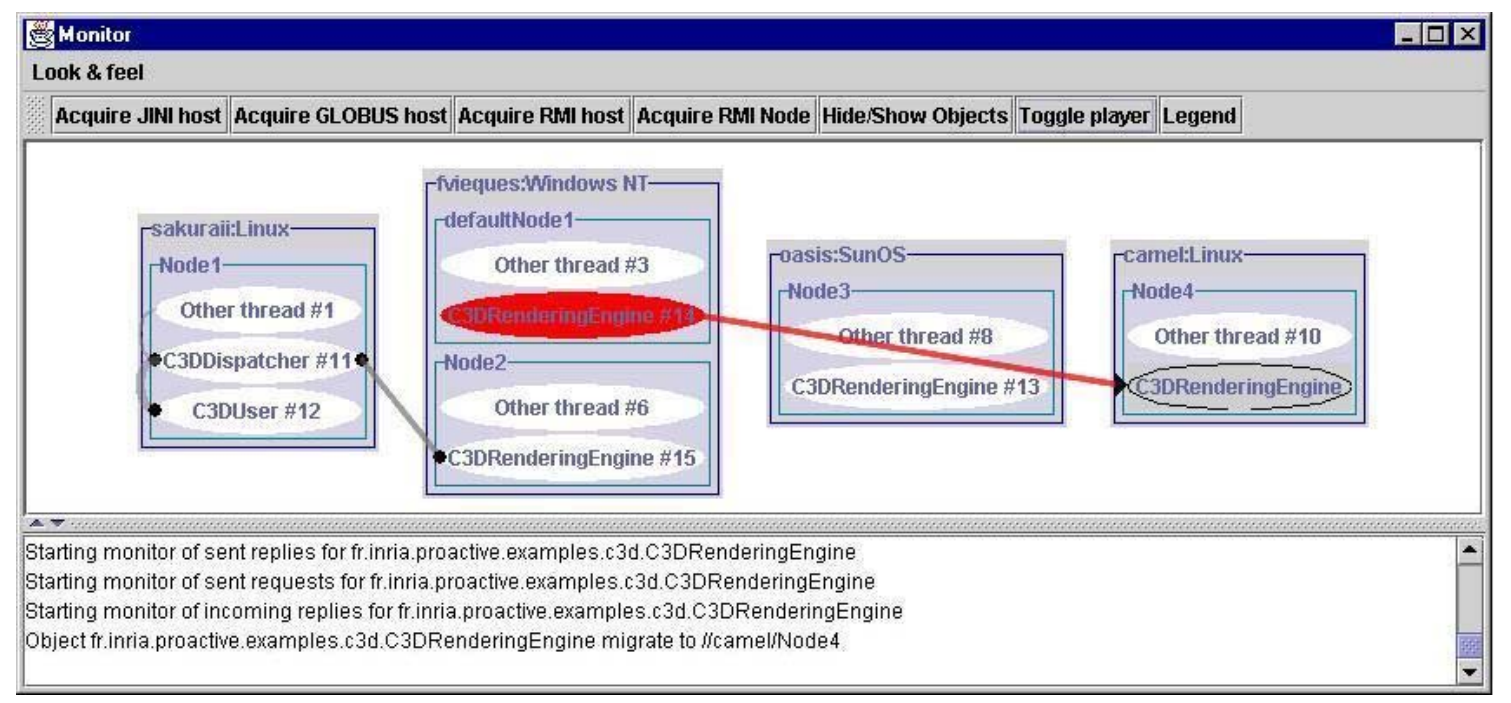

Figure 15. Drag-and-drop migration allows to graphically move objects between machines.

\section{Conclusion and Perspectives}

The work presented here focussed on reuse, flexibility, and extendability. At different levels (service routines, abstractions for control programming, libraries defining specific programming models, etc.), the system we propose tries to be both abstract with information hiding principles (black boxes simple to use), and open for customization and extension. This approach tries to give some answers to the complexity and diversity of parallel programming.

Granularity is probably another crucial point of parallel programming. In order to reach performances, a challenging task is to achieve an appropriate matching between the granularity of program activities, and the capability of the underlying parallel architecture. We believe the reusability object-oriented languages make possible to be an important answer to the problem. In particuler, $\mathrm{C}_{++} / /$makes it easy to turn an object into an active object or vice-versa, in order to adapt the granularity of program activities without not too much changes in the program.

In order to reach performances, two mechanisms have been described that can help to optimize parallel programming without too much a burden for the programmer: (1) the SharedOnRead that can help a distributed object-oriented language to be competitive with MPI, without writing large amount of code to obtain a parallel version; (2) a mechanism to overlap computations with communications in order to take advantage of pipelining in distributed object-oriented applications, without having to explicitly program the slicing of data and corresponding computations into smaller units. Both techniques apply in other distributed frameworks, such as Corba, Java RMI.

Specifically, in the framework of Java, we have defined and implemented the ProActive library [18] that offers a similar model with Java and its virtual machine. The framework being much more dynamic there are several new features that we are able to provide. First of all, it is not only possible to create remotely accessible objects, but also to "turn active" an existing object. Within the context of dynamic class loading, a JVM can receive an object for which the class was previously unknown, and the library make it possible to make this object remotely accessible. Another strong new feature is the mobility of computations. A migration primitive allows an active object to move from one machine to another, while maintaining functional all the remote references to and from itself. The graphical environment IC2D (Interactive Control and Debugging of Distribution) makes it possible to monitor and steer distributed and parallel applications. In Figure 15, the machines, the JVMs, and the active objects are graphically represented, as well as the communications that take place. Moreover, drag 
and drop migration allows to move around objects at execution, potentially from one continent to another in a metacomputing framework.

Finally, another important aspect of distributed programming is the striking correctness problems it raises. This paper didn't address them, but it is another area of investigation for our group [4,5]. We hope formal techniques, together with parallel object-oriented programming, will permit some advances in that matter.

\section{REFERENCES}

[1] Parallel Virtual Machine: a user's guide and tutorial for networked parallel computing. MIT Press (1994).

[2] MPI: The Complete Reference. MIT Press (1998).

[3] G. Agha, Actors: A Model of Concurrent Computation in Distributed Systems. MIT Press (1986).

[4] I. Attali, D. Caromel and M. Oudshoorn, A Formal Definition of the Dynamic Semantics of the Eiffel Language, in Sixteenth Australian Computer Science Conference (ACSC-16), G. Gupta, G. Mohay and R. Topor Eds., Griffith University, February (1993) 109-120.

[5] I. Attali, D. Caromel and M. Russo, Graphical Visualization of Java Objects, Threads, and Locks. IEEE Distributed Systems Online 2 (2001).

[6] H.E. Bal, M.F. Kaashoek, A.S. Tanenbaum and J. Jansen, Replication techniques for speeding up parallel applications on distributed systems. Concurrency Practice 83 Experience 4 (1992) 337-355.

[7] F. Baude, D. Caromel, N. Furmento and D. Sagnol, Optimizing Metacomputing with Communication-Computation Overlap, in 6th International Conference PaCT 2001, number 2127, V. Malyshkin Ed., LNCS, 190-204.

[8] A. Birrell, G. Nelson, S. Owicki and E. Wobber, Network Objects. Technical Report SRC-RR-115, DEC Systems Research Center (1995).

[9] G. Booch, Object-Oriented Development. IEEE Transaction on Software Engineering (1986).

[10] T. Brandes and F. Desprez, Implementing Pipelined Computation and Communication in an HPF Compiler, in Euro-Par'96, number 1123, LNCS.

[11] F. Buschmann, K. Kiefer, F. Paulish and M. Stal, The Meta-Information-Protocol: Run-Time Type Information for C++, in Proceedings of the International Workshop on Reflection and Meta-Level Architecture, A. Yonezawa and B.C. Smith Eds. (1992) 82-87.

[12] D. Caromel, Service, Asynchrony and wait-by-necessity. Journal of Object-Oriented Programming 2 (1989) $12-22$.

[13] D. Caromel, Concurrency: an Object Oriented Approach, in Technology of Object-Oriented Languages and Systems (TOOLS'90), J. Bezivin, B. Meyer and J.-M. Nerson Eds., Angkor, June (1990) 183-197.

[14] D. Caromel, Concurrency and Reusability: From Sequential to Parallel. Journal of Object-Oriented Programming 3 (1990) $34-42$.

[15] D. Caromel, Towards a Method of Object-Oriented Concurrent Programming. Communications of the ACM 36 (1993) 90-102.

[16] D. Caromel, F. Belloncle and Y. Roudier, The C $++/ /$ Language, in Parallel Programming Using C++, MIT Press (1996) $257-296$.

[17] D. Caromel, P. Dzwig, R. Kauffman, H. Liddell, A. McEwan, P. Mussi, J. Poole, M. Rigg and R. Winder, EC++ - EUROPA Parallel C++: A Draft Definition, in Proceedings of High-Performance Computing and Networking (HPCN'96), Vol. 1067, LNCS, 848-857.

[18] D. Caromel, W. Klauser and J. Vayssiere, Towards Seamless Computing and Metacomputing in Java. Concurrency Practice and Experience (1998).

[19] D. Caromel, E. Noulard and D. Sagnol, Sharedonread optimization in parallel object-oriented programming, in Computing in Object-Oriented Parallel Environments, Proceedings of ISCOPE'99, LNCS, San Francisco, Dec (1999).

[20] S. Chiba and T. Masuda, Designing an Extensible Distributed Language with Meta-Level Architecture, in Proceedings of the 7th European Conference on Object-Oriented Programming (ECOOP '93), O. Nierstrasz Ed., Springer-Verlag, Kaiserslautern, Lecture Notes in Computer Science 707 (1993) 482-501.

[21] J. Choi, J. Dongarra, S. Ostrouchov, A. Petitet, D. Walker and R.C. Whaley, A proposal for a set of parallel basic linear algebra subprograms. Technical Report Lapack Working Note 100, May (1995).

[22] A. Dave, M. Sefika and R.H. Campbell, Proxies, Application Interfaces and Distributed Systems, in proceedings of the 2nd International Workshop on Object-Orientation in Operating Systems (OOOS), Paris (France), IEEE Computer Society Press, September (1992).

[23] F. Desprez, P. Ramet and J. Roman, Optimal Grain Size Computation for Pipelined Algorithms, in Euro-Par'96, number 1123, LNCS.

[24] S. Diwan and D. Gannon, Capabilities Based Communication Model for High-Performance Distributed Applications: The Open HPC++ Approach, in IPPS/SPDP (1999). ftp://ftp.cs.indiana.edu/pub/sdiwan/capab.ps.gz

[25] I. Foster and C. Kesselman, Globus: A metacomputing infrastructure toolkit. International Journal of Supercomputer Applications 11 (1997) 115-128. 
[26] D. Gannon, S. Diwan and E. Johnson, HPC++ and the Europa Call Reification Model. ACM Applied Computing Review 4 (1996).

[27] N. Gehani, Concurrent Programming in the ADA Language: the Polling Bias. Software-Practice and Experience 14 (1984).

[28] R. Halstead, Multilisp: A Language for Concurrent Symbolic Computation. ACM Transactions on Programming Languages and Systems, October (1985)

[29] S.B. Hassen and H. Bal, Integrating task and data parallelism using shared objects, in FCRC '96: Conference proceedings of the 1996 International Conference on Supercomputing: Philadelphia, PA, USA, May 25-28, 1996, ACM Ed., ACM Press, New York (1996) 317-324.

[30] C. Hewitt, Viewing Control Structures as Patterns of Passing Messages. J. Artificial Intelligence Res. 8 (1977) $323-64$.

[31] Y. Ishikawa, A. Hori, M. Sato, M. Matsuda, J. Nolte, H. Tezuka, H. Konaka, M. Maeda and K. Kubota, Design and implementation of metalevel architecture in C++ - MPC++ approach, in Reflection'96, April (1996).

[32] G. Kiczales, J. des Rivières and D.G. Bobrow, The Art of the Metaobject Protocol. MIT Press (1991).

[33] H. Lieberman, Concurrent Object-Oriented Programming in Act 1, in Object-Oriented Concurrent Programming, A. Yonezawa and M. Tokoro Eds., MIT Press (1987).

[34] P. Madany, N. Islam, P. Kougiouris and R.H. Campbell, Practical Examples of Reification and Reflection in C++, in Proceedings of the International Workshop on Reflection and Meta-Level Architecture, A. Yonezawa and B.C. Smith Eds. (1992) $76-81$.

[35] B. Meyer, Object-Oriented Software Construction. Prentice-Hall (1988).

[36] E. Noulard, N. Emad and L. Flandrin, Calcul numérique parallèle et technologies objet. Technical Report Rapport PRISM 1998/003, ADULIS/PRiSM, Juillet (1997). Révision du 30/01/98.

[37] R. Rashid, R. Baron, A. Forin, D. Golub, M. Jones, D. Orr and R. Sanzi, Mach: a foundation for open systems (operating systems), in Workstation Operating Systems: Proceedings of the Second Workshop on Workstation Operating Systems (WWOS-II), Pacific Grove, CA, USA, September 27-29, 1989, IEEE Ed., IEEE Computer Society Presspages (1989) 109-113.

[38] Y. Saad, Iterative Methods for Sparse Linear Systems. PWS Publishing Company, New York (1996).

[39] M. Shapiro, Structure and Encapsulation in Distributed Systems: the Proxy Principle, in Proceedings of the 6th International Conference on Distributed Computing Systems, Cambridge, MA, USA, IEEE, May (1986) 198-204.

[40] C.W. Tseng, An Optimizing Fortran D Compiler for MIMD Distributed-Memory Machines. Ph.D. thesis, Rice University (1993).

[41] G. Wilson and P. Lu Eds., Parallel Programming Using C++. MIT Press (1996).

[42] Y. Yokote and M. Tokoro, Concurrent Programming in ConcurrentSmalltalk, in Object-Oriented Concurrent Programming, A. Yonezawa and M. Tokoro Eds., MIT Press (1987).

[43] A. Yonezawa, E. Shibayama, T. Takada and Y. Honda, Modelling and Programming in an Object-Oriented Concurrent Language ABCL/1, in Object-Oriented Concurrent Programming, A. Yonezawa and M. Tokoro Eds., MIT Press (1987).

To access this journal online:

www.edpsciences.org 